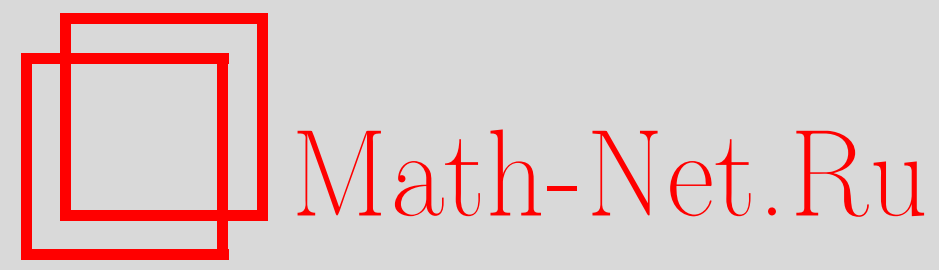

Н. П. Салихов, Об одном обобщении расстояния Чернова, Теория вероятн. и ее примен., 1998, том 43, выпуск 2, 294314

DOI: https://doi.org/10.4213/tvp1466

Использование Общероссийского математического портала Math-Net.Ru подразумевает, что вы прочитали и согласны с пользовательским соглашением http://www. mathnet.ru/rus/agreement

Параметры загрузки:

IP : 54.210 .77 .194

26 апреля 2023 г., 09:33:29

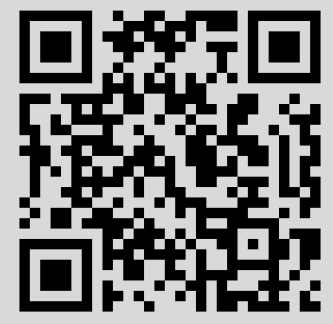




\section{ОБ ОДНОМ ОБОБЩЕНИИ РАССТОЯНИЯ ЧЕРНОВА}

Вводится величина $\rho(\mathbf{p} ; A, B)$, характеризующая при заданном векторе p расстояние между конечными множествами $A, B$ векторов вероятностей исходов в полиномиальных схемах испытаний с общим множеством исходов. В случае одноэлементных $A=\{\mathbf{a}\}, B=\{\mathbf{p}\}$ величина $\rho(\mathbf{p} ; A, B)$ совпадает с расстоянием Чернова между $\mathbf{p}$ и а. Указан вероятностный смысл и найдены некоторые свойства обобщенного расстояния Чернова $\rho(\mathbf{p} ; A, B)$. Для различения $m$ полиномиальных распределений с параметрами $\left(n, \mathbf{p}_{1}\right), \ldots,\left(n, \mathbf{p}_{m}\right)$ рассмотрено байесовское решаюшее правило, в котором истинное распределение указывается в $k \in\{1, \ldots, m-1\}$ наиболее правдоподобных вариантах. Для этого правила найдены явные и асимптотические (при $n \rightarrow \infty$ ) оценки вероятностей ошибок, зависящие от не более $C_{m-1}^{k}$ обобщенных расстояний Чернова, доказана его оптимальность в некотором смысле.

Ключевые слова и фразы: полиномиальная схема испытаний, расстояние Кульбака-Лейблера, расстояние Чернова, различение нескольких простых гипотез, байесовское решающее правило, оценки вероятностей ошибок.

\section{1. Введение}

Цель работы - указать «содержательный статистический смысл» (выражение из [1, §4]) величины, которая естественным образом обобшает расстояние Чернова (см. [2]) между двумя распределениями, на примере задачи о различении нескольких полиномиальных распределений.

Уточним постановку этой задачи. Пусть натуральное $r \geqslant 2$,

$$
\begin{aligned}
\Omega & =\left\{\mathbf{u}=\left(u_{1}, \ldots, u_{r}\right): u_{1} \geqslant 0, \ldots, u_{r} \geqslant 0, u_{1}+\cdots+u_{r}=1\right\} \\
\Omega_{+} & =\left\{\mathbf{u}=\left(u_{1}, \ldots, u_{r}\right): u_{1}>0, \ldots, u_{r}>0, u_{1}+\cdots+u_{r}=1\right\} .
\end{aligned}
$$

Пусть проведено $n$ независимых испытаний по полиномиальной схеме $H(\mathbf{p})$ с $r$ исходами; $p_{1}, \ldots, p_{r}-$ вероятности исходов, $\mathbf{p}=\left(p_{1}, \ldots\right.$, $\left.p_{r}\right) \in \Omega_{+}, x_{k}$ - число появлений $k$-го $(k=1, \ldots, r)$ исхода, $\Omega_{n}-$ множество всех элементарных событий, $\left|\Omega_{n}\right|=C_{n+r-1}^{r-1}, \underline{\mathbf{x}}=\left(\underline{x}_{1}, \ldots, \underline{x}_{r}\right)=$

*ФАПСИ, Б. Кисельный пер., 4, 103031 Москва, Россия. 
$\left(x_{1} / n, \ldots, x_{r} / n\right)$. Тогда $\mathbf{P}_{n}(\underline{\mathbf{x}}, \mathbf{p})=\left(n ! /\left(x_{1} ! \cdots x_{r} !\right)\right) p_{1}^{x_{1}} \cdots p_{r}^{x_{r}}-$ вероя $\mathbf{T}^{-}$ ность (правдоподобие) элементарного события ‥ Будем говорить, что гипотеза $H(\mathbf{p}, n)$ верна, если $n$ независимых испытаний проведены по схеме $H(\mathbf{p})$.

Пусть имеются $m$ альтернативных гипотез $H\left(\mathbf{p}_{j}, n\right), \mathbf{p}_{j}=\left(p_{j, 1}, \ldots\right.$, $\left.p_{j, r}\right), j=1, \ldots, m$, где $\mathbf{p}_{1}, \ldots, \mathbf{p}_{m}$ - различные элементы из $\Omega_{+}$, множество исходов одно и то же при всех $m$ гипотезах. Известную задачу множественной классификации $[3$, гл. $6, \S 4]$ или идентификации $[4, \S 7 \mathrm{~d}]$ для указанных $m$ гипотез можно сформулировать следующим образом. Требуется построить при каждом $n$ правило, по которому статистик по наблюдению $\underline{\mathbf{x}}$, может сделать однозначный вывод: гипотеза $H\left(\mathbf{p}_{i}, n\right)$ верна. Такое правило будем характеризовать вероятностями $\alpha_{t, n}$ отвергнуть истинную гипотезу $H\left(\mathbf{p}_{t}, n\right)$ (т.е. сделать вывод: верна одна из гипотез $\left.H\left(\mathbf{p}_{i}, n\right), i \neq t\right)$.

Известно, что для любой последовательности правил

$$
\liminf _{n \rightarrow \infty} n^{-1} \ln \max _{1 \leqslant t \leqslant m} \alpha_{t, n} \geqslant-\rho
$$

где $\rho$ - минимум $m(m-1)$ расстояний Чернова между $\mathbf{p}_{i}, \mathbf{p}_{j}, i, j \in$ $\{1, \ldots, m\}, i \neq j$, причем существует оптимальная последовательность (байесовских) правил, для которой

$$
\lim _{n \rightarrow \infty} n^{-1} \ln \max _{1 \leqslant t \leqslant m} \alpha_{t, n}=-\rho
$$

(см. [5]). При малых $n$, получаемая из (2), оценка для $\max _{1 \leqslant t \leqslant m} \alpha_{t, n}$ может оказаться неудовлетворительной. Чтобы не отвергнуть при малом $n$ истинную гипотезу, в данной работе предлагается вместо однозначного вывода делать по $\underline{\mathbf{x}}$ многозначный вывод: верна одна из гипотез множества $d(\underline{\mathbf{x}})=\left\{H\left(\mathbf{p}_{i_{1}}, n\right), \ldots, H\left(\mathbf{p}_{i_{k}}, n\right)\right\}, i_{1}, \ldots, i_{k}$ - различные элементы множества $\{1, \ldots, m\}, 1 \leqslant k \leqslant m-1 ;$ при $k=1$ вывод однозначный. Для такого решающего правила вероятность $\alpha_{t, n}$ - это вероятность того, что истинная гипотеза $H\left(\mathbf{p}_{t}, n\right)$ не входит в $d(\underline{\mathbf{x}})$. Оказывается, что в этом случае (при $k>1$ ) верно неравенство (1), а при определенных ограничениях на векторы $\mathbf{p}_{t}$ справедливо и равенство (2), в которых $\rho$ заменяется на минимум не более $m C_{m-1}^{k}$ обобщенных расстояний Чернова. Правила $D_{B}(n ; k)$, для которых верно (2), задаются множествами $d(\underline{\mathbf{x}})$, где $i_{1}, \ldots, i_{k}$ - номера распределений с максимальными правдоподобиями наблюдения $\underline{\mathbf{x}}$ (см. теорему 7 , замечание 8). Для правил $D_{B}(n ; k)$ получены также явные верхние оценки вероятностей $\alpha_{t, n}=\alpha_{t}\left(D_{B}(n ; k)\right)$, зависяшие от обобщенных расстояний Чернова (см. теорему 6) и от расстояний Чернова (см. теорему 8).

При решении классической задачи об $r$-гранной игральной кости, когда $\varepsilon>1 / r$ - вероятность выпадения одной из граней (фальшивой), $\delta=(1-\varepsilon) /(r-1)$ - вероятность выпадения каждой из остальных граней (см. $[3$, гл. $6, \S 4],[6$, примечание к $\S 1]$ ), предложенный метоп (при 
различении $r$ альтернативных гипотез о фальшивой грани) дает следуюшие результаты (см. примеры 1,3 ). Все требуюшиеся для вычисления $\rho$ обобщенные расстояния Чернова одинаковы и равны

$$
\rho_{k}^{*}=-\ln \left(\delta\left(r-k-1+(k+1)\left(\frac{\varepsilon}{\delta}\right)^{1 /(k+1)}\right)\right)
$$

(следовательно, $\rho=\rho_{k}^{*}$ ). При применении правила $D_{B}(n ; k)=\{$ фальшивая грань - одна из $k$ самых часто выпавших при $n$ бросаниях $\}$ для вероятностей ошибок верны оценки

$$
\alpha_{t, n} \leqslant C_{r-1}^{k} \exp \left(-n \rho_{k}^{*}\right), \quad \lim _{n \rightarrow \infty} n^{-1} \ln \alpha_{t, n}=-\rho_{k}^{*}
$$

при $t=1, \ldots, n$. Формулы (3), (4) подтверждают интуитивно ясную закономерность - чем больше $k$ и $\varepsilon$, тем больше вероятность того, что фальшивая грань - одна из $k$ самых часто выпавших при $n$ бросаниях ( $\rho_{k}^{*}$ возрастает с ростом $k$ и $\varepsilon$ ).

Далее используем следуюшие обозначения: $\chi(A)$ - индикатор события $A$;

$$
I(\mathbf{u}, \mathbf{p})=\sum_{k=1}^{r} u_{k} \ln \frac{u_{k}}{p_{k}}
$$

$\left(u_{i} \ln \left(u_{i} / p_{i}\right)=0\right.$ при $\left.u_{i}=0\right)-$ расстояние Кульбака-Лейблера от $\mathbf{u} \in \Omega$ до $\mathbf{p} \in \Omega_{+}$(см. $[7$, гл. $\left.6, \S 2]\right) ; \mathbf{P}_{n}(\omega, \mathbf{p})=\sum_{\mathbf{x} \in \omega \cap \Omega_{n}} \mathbf{P}_{n}(\underline{\mathbf{x}}, \mathbf{p})$ для $\mathbf{p} \in \Omega_{+}$ и $\omega \subseteq \Omega$.

Множество $\Omega$ будем рассматривать как метрическое пространство c расстоянием $d(\mathbf{x}, \mathbf{y})=\left(\sum_{i=1}^{r}\left(x_{i}-y_{i}\right)^{2}\right)^{1 / 2}$ для $\mathbf{x}, \mathbf{y} \in \Omega$. Пусть $[\omega]-$ замыкание множества $\omega \subseteq \Omega$,

$$
\begin{array}{r}
M(\mathbf{a}, \mathbf{b})=\left\{\mathbf{u}=\left(u_{1}, \ldots, u_{r}\right): \mathbf{u} \in \Omega, \sum_{k=1}^{r} u_{k} \ln \frac{a_{k}}{b_{k}} \geqslant 0\right\} \\
M_{+}(\mathbf{a}, \mathbf{b})=\left\{\mathbf{u}=\left(u_{1}, \ldots, u_{r}\right): \mathbf{u} \in \Omega, \sum_{k=1}^{r} u_{k} \ln \frac{a_{k}}{b_{k}}>0\right\}
\end{array}
$$

для $\mathbf{a}, \mathbf{b} \in \Omega_{+}$. Для $J \subseteq\{1, \ldots, r\}$ положим

$$
\Omega(J)=\left\{\mathbf{u} \in \Omega: u_{j}>0 \text { для } j \in J, u_{j}=0 \text { для } j \in\{1, \ldots, r\} \backslash J\right\} .
$$

Будем использовать следуюцие просто доказываемые утверждения.

а) Если $\omega \subseteq \Omega-$ выпуклое множество (при $|\omega|=1$ множество $\omega$ считается замкнутым и выпуклым), то существует единственное множество $J=J(\omega) \subseteq\{1, \ldots, r\}$ такое, что $\omega \subseteq[\Omega(J)], \omega \cap \Omega(J) \neq \varnothing$, $|J|=s(\omega)=\sup _{u \in \omega} \sum_{k=1}^{r} \chi\left(u_{k}>0\right) \geqslant 1$.

б) Если $\omega=\left\{\mathbf{u} \in \Omega: f_{1}(\mathbf{u})>0, \ldots, f_{t}(\mathbf{u})>0\right\} \neq \varnothing, f_{1}(\mathbf{u}), \ldots$, $f_{t}(\mathbf{u})$ - непрерывные на $\Omega$ функции, то $\omega \cap \Omega_{+} \neq \varnothing$ и $\omega$ - открытое в $\Omega$ множество. 
в) Если $g_{1}(\mathbf{u}), \ldots, g_{t}(\mathbf{u})$ - непрерывные и вогнутые на $\Omega$ функции, $\omega_{+}=\left\{\mathbf{u} \in \Omega: g_{1}(\mathbf{u})>0, \ldots, g_{t}(\mathbf{u})>0\right\} \neq \varnothing$, то $\omega_{+}$- выпуклое множество, $\left[\omega_{+}\right]=\omega=\left\{\mathbf{u} \in \Omega: g_{1}(\mathbf{u}) \geqslant 0, \ldots, g_{t}(\mathbf{u}) \geqslant 0\right\}$.

г) Если $x_{i, n}>0$ для $i=1, \ldots, t, n=1,2, \ldots, \lim _{n \rightarrow \infty} n^{-1} \ln x_{i, n}=$ $-b_{i}$ для $i=1, \ldots, t$, то

$$
\lim _{n \rightarrow \infty} n^{-1} \ln \max _{1 \leqslant i \leqslant t} x_{i, n}=\lim _{n \rightarrow \infty} n^{-1} \ln \left(x_{1, n}+\cdots+x_{t, n}\right)=-\min _{1 \leqslant i \leqslant t} b_{i} .
$$

\section{2. Обобщенное расстояние Чернова}

В п. 2,3 предполагается, что $A, B$ - конечные, непустые подмножества множества $\Omega_{+}, \mathbf{p} \in \Omega_{+}$.

Пусть $M(\mathbf{a}, B)=\bigcap_{b \in B} M(\mathbf{a}, \mathbf{b}), M(A, \mathbf{b})=\bigcap_{a \in A} M(\mathbf{a}, \mathbf{b}), M(A, B)=$ $\mathrm{\cap}_{a \in A} M(\mathbf{a}, B)$ и аналогично (с заменой $M$ на $M_{+}$) определяются множества $M_{+}(\mathbf{a}, B), M_{+}(A, \mathbf{b}), M_{+}(A, B)$.

При $M(A, B) \neq \varnothing$ назовем величину $\rho(\mathbf{p} ; A, B)=\min _{u \in M(A, B)} I(\mathbf{u}, \mathbf{p})$ обобщенным расстоянием Чернова от $A$ до $B$ при заданном $\mathbf{p}$.

Укажем следуюшие очевидные свойства величины $\rho(\mathbf{p} ; A, B)$ :

1) $\rho(\mathbf{p} ; A, B) \geqslant 0$ и $\rho(\mathbf{p} ; A, B)=0$ только при $\mathbf{p} \in M(A ; B)$ (следствие свойств расстояния Кульбака-Лейблера, $[7$, гл. 6, § 2]);

2) $\rho(\mathbf{p} ; \mathbf{a}, \mathbf{b}) \rho(\mathbf{p} ; \mathbf{b}, \mathbf{a})=0$

3) $\rho(\mathbf{p} ; \mathbf{a}, B) \leqslant I(\mathbf{a}, \mathbf{p})$;

4) $\rho(\mathbf{p} ; A, B) \geqslant \max _{(a, b) \in A \times B} \rho(\mathbf{p} ; \mathbf{a}, \mathbf{b})$;

5) $\rho(\mathbf{p} ; C, D) \leqslant \rho(\mathbf{p} ; A, B)$, если $C \times D \subseteq A \times B$.

Пусть $\sigma_{i}=\prod_{(a, b) \in A \times B}\left(a_{i} / b_{i}\right)^{\sigma_{a, b}}, c_{i}=p_{i} \sigma_{i} / \sigma$ для $i=1, \ldots, r, \sigma=$ $\sum_{i=1}^{r} p_{i} \sigma_{i}, \mathbf{c}=\left(c_{1}, \ldots, c_{r}\right)$. Легко проверить, что если все $\sigma_{a, b} \geqslant 0$ и $\sigma \leqslant 1\left(\right.$ все $\sigma_{a, b} \leqslant 0$ и $\left.\sigma \geqslant 1\right)$, то $I(\mathbf{u}, \mathbf{c}) \leqslant I(\mathbf{u}, \mathbf{p})(I(\mathbf{u}, \mathbf{c}) \geqslant I(\mathbf{u}, \mathbf{p}))$ при любом и $\in M(A, B)$ и, следовательно, $\rho(\mathbf{c} ; A, B) \leqslant \rho(\mathbf{p} ; A, B)(\rho(\mathbf{c} ; A, B) \geqslant$ $\rho(\mathbf{p} ; A, B))$. Поэтому имеем $\rho(\mathbf{a} ; A, B) \leqslant \rho(\mathbf{p} ; A, B)$ при $\mathbf{p} \in B, \mathbf{a} \in A$ $(\rho(\mathbf{b} ; A, B) \geqslant \rho(\mathbf{p} ; A, B)$ при $\mathbf{p} \in A, \mathbf{b} \in B)$.

Следуюшая теорема указывает вероятностный смысл величины $\rho(\mathbf{p} ; A, B)$.

Tеорема 1. Ecлu $M_{+}(A, B) \neq \varnothing, M_{+}(A, B) \subseteq \omega \subseteq M(A, B)$, mo $\mathbf{P}_{n}(\omega, \mathbf{p})>0$ при всех достаточно больиих $n u$

$$
\lim _{n \rightarrow \infty} n^{-1} \ln \mathbf{P}_{n}(\omega, \mathbf{p})=-\rho(\mathbf{p} ; A, B) \text {. }
$$

Лемма 1. Пусть $\omega \subseteq \Omega, \omega \cap \Omega_{n} \neq \varnothing$ при всех достаточно больwux $n u$

$$
\lim _{n \rightarrow \infty} \min _{u \in \omega \cap \Omega_{n}} I(\mathbf{u}, \mathbf{p})=\min _{u \in[\omega]} I(\mathbf{u}, \mathbf{p}) .
$$

Toгda $\lim _{n \rightarrow \infty} n^{-1} \ln \mathbf{P}_{n}(\omega, \mathbf{p})=-\min _{u \in[\omega]} I(\mathbf{u}, \mathbf{p})$.

Эта лемма - простое следствие оценки

$$
C_{r} n^{-(r-1) / 2} \leqslant \mathbf{P}_{n}(\omega, \mathbf{p}) \exp \left(n \min _{u \in \omega \cap \Omega_{n}} I(\mathbf{u}, \mathbf{p})\right) \leqslant C_{n+r-1}^{r-1},
$$


которая верна, с некоторой положительной константой $C_{r}$, если $n \geqslant r$ и $\omega \cap \Omega_{n} \neq \varnothing$. В [8] (см. теорему 2.1) без доказательства утверждается, что можно положить $C_{\tau}=\frac{1}{2}$. Уточняя оценку $(8.86)$ в $[9$, гл. $8, \S 4]$, можно получить значение

$$
C_{r}=\sqrt{2 \pi}\left(\frac{r}{2 \pi e^{1 / 6}}\right)^{r / 2}
$$

Для $\omega=M(\mathbf{a}, \mathbf{b})$ равенство (5) следует из леммы А2 работы [8]. Из лемм 2,3 и из того, что множество $\omega=M_{+}(A, B) \neq \varnothing$ открыто в $\Omega$ и $\left[M_{+}(A, B)\right]=M(A, B)$ (см. утверждения б), в)), следует $(5)$ для $\omega=M_{+}(A, B) \neq \varnothing$, что и доказывает теорему 1 .

Лемма 2. Пусть $\omega \subseteq \Omega, \omega \neq \varnothing, \min _{u \in[\omega]} I(\mathbf{u}, \mathbf{p})=I(\mathbf{y}, \mathbf{p})$ nри некотором $\mathbf{y} \in[\omega]$ и существует последовательность $\mathbf{u}_{n}, n=1,2, \ldots$, сходяцаяся $\kappa$ у $u$ такая, что $u_{n} \in \omega \cap \Omega_{n}$ при всех достаточно больших $n$. Тогда верно равенство (5).

Д ок азате л в с в о. Достаточно учесть, что

$$
\lim _{n \rightarrow \infty} I\left(\mathbf{u}_{n}, \mathbf{p}\right)=I(\mathbf{y}, \mathbf{p}) \quad \text { и } \quad \min _{u \in[\omega]} I(\mathbf{u}, \mathbf{p}) \leqslant \min _{u \in \omega \cap \Omega_{n}} I(\mathbf{u}, \mathbf{p}) \leqslant I\left(\mathbf{u}_{n}, \mathbf{p}\right)
$$

при всех достаточно больших $n$.

Лемма 3. Пусть $\omega \subseteq \Omega$ - непустое отхрытое в $\Omega$ множество. Тогда для любой точки $y \in[\omega]$ существует последовательность $\mathbf{u}_{n}$, $n=1,2, \ldots$, сходячияся $\propto$ у $u$ maкая, что $\mathbf{u}_{n} \in \omega \cap \Omega_{n}$ nри всех достаточно больиих $n$.

Д ок а 3 а т е л ь с т о. Пусть $n_{0}=0, \delta_{1}, \delta_{2}, \ldots$ - убывающая и сходящаяся к 0 последовательность положительных чисел. Для $m=$ $1,2, \ldots$ в $\delta_{m}$-окрестности точки у найдем точку $\mathbf{z}_{m}=\left(z_{m, 1}, \ldots, z_{m, r}\right)$ множества $\omega$. При некотором $\varepsilon_{m} \in\left(0, \delta_{m}\right)$ множество $\left\{\mathbf{u}: \mathbf{u} \in \Omega, d\left(\mathbf{z}_{m}, \mathbf{u}\right)<\right.$ $\left.\varepsilon_{m}\right\} \subseteq \omega$. Пусть $v_{n, m}=\left(v_{n, m, 1}, \ldots, v_{n, m, r}\right) \in \Omega$, где

$$
\begin{aligned}
& v_{n, m, t}=\left(n-\sum_{j \in\{1, \ldots, r\} \backslash\{t\}}\left[n z_{m, j}\right]\right) n^{-1} \text { при некотором } t \in\{1, \ldots, r\}, \\
& v_{n, m, j}=\frac{\left[n z_{m, j}\right]}{n} \text { для } j \in\{1, \ldots, r\} \backslash\{t\} .
\end{aligned}
$$

Torда

$$
\begin{aligned}
d\left(\mathbf{z}_{m}, \mathbf{v}_{n, m}\right) & <\frac{\sqrt{r(r-1)}}{n}<\varepsilon_{m}, \\
d\left(\mathbf{y}, \mathbf{v}_{n, m}\right) & \leqslant d\left(\mathbf{y}, \mathbf{z}_{m}\right)+d\left(\mathbf{z}_{m}, \mathbf{v}_{n, m}\right)<\delta_{m}+\varepsilon_{m}<2 \delta_{m}
\end{aligned}
$$

при всех $n \geqslant n_{m}=1+\max \left(n_{m-1},\left[r / \varepsilon_{m}\right]\right)$.

Для последовательности $\left\{\mathbf{u}_{n}\right\}$, где $\mathbf{u}_{n}=\mathbf{v}_{n, m}$ при $n=n_{m}, \ldots$, $n_{m+1}-1, m=1,2, \ldots$, верно утверждение теоремы. 
3 а м е ч а н и е 1. Если $A \cap B \neq \varnothing$, то $M_{+}(A, B)=\varnothing$. Если $A \cap B=$ $\varnothing,|A|=1$, то $A \subseteq M_{+}(A, B), M_{+}(A, B) \neq \varnothing$. Пусть $A \cap B=\varnothing,|A|>$ $1, M(A, B) \neq \varnothing, M_{+}(A, B)=\varnothing$. Тогда теорему 1 нельзя применить для $\omega \subseteq M(A, B), \omega \neq \varnothing$. Однако во многих случаях оказывается, что $M(A, B) \cap \Omega_{n}=\varnothing\left(\right.$ т.e. $\left.\mathbf{P}_{n}(\omega, \mathbf{p})=0\right)$ при всех достаточно больших $n$. Действительно, пусть

$$
s=\sup _{\mathbf{u} \in M(A, B)} \sum_{(a, b) \in A \times B} \chi\left(\sum_{i=1}^{r} u_{i} \ln \frac{a_{i}}{b_{i}}>0\right)<|A| \cdot|B| .
$$

Из выпуклости $M(A, B)$ следует сушествование единственного множества

$$
M=\left\{(\mathbf{a}, \mathbf{b}) \in A \times B: \sum_{i=1}^{r} u_{i} \ln \frac{a_{i}}{b_{i}}=0 \text { для всех } \mathbf{u} \in M(A, B)\right\},
$$

$|M|=|A| \cdot|B|-s$, такого, что при $s \geqslant 1$ величина $\sum_{i=1}^{r} v_{i} \ln \left(a_{i} / b_{i}\right)>0$ при некотором $\mathbf{v}=\left(v_{1}, \ldots, v_{r}\right) \in M(A, B)$ для всех $(\mathbf{a}, \mathbf{b}) \in A \times B \backslash M$. При этом $|\{\mathbf{a}:(\mathbf{a}, \mathbf{b}) \in M\}|>1$ (если $\mathbf{a}-$ единственный элемент в $\{\mathbf{a}:(\mathbf{a}, \mathbf{b}) \in M\}$, то при малом $\left.\varepsilon>0(\mathbf{v}+\varepsilon \mathbf{a}) /(1+\varepsilon) \in M_{+}(A, B)\right)$. Таким образом, условие $M(A, B) \cap \Omega_{n} \neq \varnothing$ при всех $n \geqslant n_{0}$ приводит к тому, что в $A \times B$ существуют две пары $\left(\mathbf{a}_{1}, \mathbf{b}_{1}\right),\left(\mathbf{a}_{2}, \mathbf{b}_{2}\right), \mathbf{a}_{1} \neq \mathbf{a}_{2}$, для которых при кажцом $n \geqslant n_{0}$ найдутся целые неотрицательные числа $x_{1}, \ldots, x_{r}$, удовлетворяюшие равенствам

$$
x_{1}+\cdots+x_{r}=n, \quad \prod_{i=1}^{r} a_{j, i}^{x_{i}}=\prod_{i=1}^{r} b_{j, i}^{x_{i}}, \quad j=1,2 .
$$

Если координаты всех $\mathbf{a} \in A, \mathbf{b} \in B$ рациональны, то проверка последних равенств представляет собой теоретико-числовую задачу. Из этих равенств также следует (см. критерий Вороного [10, гл. $2, \S 3])$ несовместность (легко решаемой) системы неравенств

$$
\begin{gathered}
y_{1} \ln \frac{a_{1, i}}{b_{1, i}}+y_{2} \ln \frac{a_{2, i}}{b_{2, i}}<0, \\
i \in I, \quad I=\left\{i \in\{1, \ldots, r\}:\left|a_{1, i}-b_{1, i}\right|+\left|a_{2, i}-b_{2, i}\right|>0\right\} .
\end{gathered}
$$

Вероятностный смысл величины $\rho(\mathbf{p} ; A, B)$ можно дополнить неравенством $\mathbf{P}_{n}(M(A, B), \mathbf{p}) \leqslant \exp (-n \rho(\mathbf{p} ; A, B))$. Это неравенство получается из следующих леммы и теоремы.

Лемма 4. Пусть $\omega \subseteq \Omega-$ замкнутое выпуклое множество, $\mathbf{p} \in$ $\Omega_{+} \mid \omega, J=J(\omega)$. Тогда минимум функции $I(\mathbf{u}, \mathbf{p})$ на $\omega$ достигается $в$ такой точке $\mathbf{z} \in \Omega(J)$, ито $(1-\varepsilon) \mathbf{z}+\varepsilon \mathbf{p} \notin \omega$ при любом $\varepsilon \in(0,1)$. 
Д ок а за т е л в с т в о. Случай $|\omega|=1$ очевиден. Пусть $|\omega|>1$. Если $(1-\varepsilon) \mathbf{z}+\varepsilon \mathbf{p} \in \omega, \varepsilon \in(0,1)$, то (используя строгую выпуклость функции $I(\mathbf{u}, \mathbf{p}))$ имеем:

$$
\begin{aligned}
& I((1-\varepsilon) \mathbf{z}+\varepsilon \mathbf{p}, \mathbf{p})<(1-\varepsilon) I(\mathbf{z}, \mathbf{p})+\varepsilon I(\mathbf{p}, \mathbf{p})=(1-\varepsilon) I(\mathbf{z}, \mathbf{p}), \\
& I((1-\varepsilon) \mathbf{z}+\varepsilon \mathbf{p}, \mathbf{p})-I(\mathbf{z}, \mathbf{p})<-\varepsilon I(\mathbf{z}, \mathbf{p})<0 .
\end{aligned}
$$

Но тогда $\mathbf{z}$ не является точкой минимума на $\omega$. Следовательно, $(1-$ $\varepsilon) \mathbf{z}+\varepsilon \mathbf{p} \notin \omega$. Сушествует $\mathbf{w}=\left(w_{1}, \ldots, w_{r}\right) \in \omega \cap \Omega(J), \mathbf{w} \neq \mathbf{z}(\mathbf{c m}$. утверждение а)). По теореме Лагранжа

$$
I((1-\varepsilon) \mathbf{z}+\varepsilon \mathbf{w}, \mathbf{p})-I(\mathbf{z}, \mathbf{p})=\varepsilon \sum_{k \in J}\left(w_{k}-z_{k}\right) \ln \frac{z_{k}+\delta\left(w_{k}-z_{k}\right)}{p_{k}}
$$

при некотором $\delta \in(0, \varepsilon)$ для любого $\varepsilon \in(0,1)$. При $\mathbf{z} \in \omega \backslash \Omega(J)$ и при достаточно малом $\varepsilon \in(0,1)$ правая часть в $(6)$ отрицательна, что приводит (с учетом выпуклости $\omega$ ) к противоречию:

$$
(1-\varepsilon) \mathbf{z}+\varepsilon \mathbf{w} \in \omega, \quad I((1-\varepsilon) \mathbf{z}+\varepsilon \mathbf{w}, \mathbf{p})<I(\mathbf{z}, \mathbf{p}) .
$$

Следовательно, $\mathbf{z} \in \Omega(J)$.

Теорема 2. При выполнении условий леммы 4

$$
\mathbb{P}_{n}(\omega, \mathbf{p}) \leqslant \exp \left(-n \min _{u \in \omega} I(\mathbf{u}, \mathbf{p})\right) .
$$

Д ок аз а те ль с тв о. Пусть точка z удовлетворяет условиям леммы 4. Тогда

$$
\begin{aligned}
\mathbf{P}_{n}(\omega, \mathbf{p}) & =\sum_{\left(n_{1}, \ldots, n_{r}\right) / n \in \omega} \frac{n !}{n_{1} ! \cdots n_{r} !} z_{1}^{n_{1}} \cdots z_{r}^{n_{r}} \exp \left(-n \sum_{k=1}^{r} \frac{n_{k}}{n} \ln \frac{z_{k}}{p_{k}}\right) \\
& \leqslant \exp \left(-n \min _{u \in \omega} f(\mathbf{u})\right),
\end{aligned}
$$

где $f(\mathbf{u})=\sum_{k=1}^{r} u_{k} \ln \left(z_{k} / p_{k}\right)$. Из неравенства $f(\mathbf{u}) \leqslant I(\mathbf{u}, \mathbf{p})$ (равносильного неравенству $I(\mathbf{u}, \mathbf{z}) \geqslant 0)$ следует, что $\min _{u \in \omega} f(\mathbf{u}) \leqslant I(\mathbf{z}, \mathbf{p})$. Достаточно доказать, что

$$
\min _{u \in \omega} f(\mathbf{u})=I(\mathbf{z}, \mathbf{p})
$$

Пусть $|\omega|>1$ (случай $|\omega|=1$ очевиден). Допустим противное; тогда $f(\mathbf{w})<I(\mathbf{z}, \mathbf{p})$ при некотором $w \in \omega$. По формуле Тейлора при $\varepsilon \in(0,1)$ и некотором $\delta \in(0, \varepsilon)$

$$
\begin{aligned}
& I((1-\varepsilon) \mathbf{z}+\varepsilon \mathbf{w}, \mathbf{p})-I(\mathbf{z}, \mathbf{p}) \\
& \quad=\varepsilon(f(\mathbf{w})-I(\mathbf{z}, \mathbf{p}))+2^{-1} \varepsilon^{2} \sum_{k \in J} \frac{\left(w_{k}-z_{k}\right)^{2}}{z_{k}+\delta\left(w_{k}-z_{k}\right)} .
\end{aligned}
$$


Эта величина при достаточно малом $\varepsilon$ отрицательна, что приводит к противоречию (7).

Теорема 3. IIycmb

$$
|M(A, B)|>1, \quad J=J(M(A, B)) \quad \text { u } \quad \mathbf{p} \in \Omega_{+} \backslash M(A, B) .
$$

Для того итобы $\rho(\mathbf{p} ; A, B)=I(\mathbf{z}, \mathbf{p})$ при некотором $\mathbf{z}=\left(z_{1}, \ldots, z_{r}\right) \in$ $M(A, B)$, необходимо и достаточно, чтобы выполнялись условия:

$$
\begin{aligned}
& \mathbf{z} \in \Omega(J), \quad z_{t}=p_{t} \sigma_{t}\left(\sum_{j \in J} p_{j} \sigma_{j}\right)^{-1} \quad \partial \Omega s t \in J, \\
& \sum_{t \in J} p_{t} \sigma_{t} \ln \sigma_{t}=0, \quad \sum_{t \in J} p_{t} \sigma_{t} \ln \frac{a_{t}}{b_{t}} \geqslant 0
\end{aligned}
$$

для всех nap $(\mathbf{a}, \mathbf{b}) \in A \times B, \mathbf{a}=\left(a_{1}, \ldots, a_{r}\right), \mathbf{b}=\left(b_{1}, \ldots, b_{r}\right)$, где

$$
\sigma_{t}=\exp \left(\sum_{(a, b) \in A \times B} \sigma_{a, b} \ln \frac{a_{t}}{b_{t}}\right), \quad \sigma_{a, b} \geqslant 0
$$

$\partial \bumpeq$ в вех $\operatorname{nap}(\mathbf{a}, \mathbf{b}) \in A \times B$.

Д о к а з а т е л с с т о. Так как $M(A, B) \subseteq[\Omega(J)]$, то

$$
\rho(\mathbf{p} ; A, B)=\min _{u \in M(A, B)} \sum_{t \in J} u_{t} \ln \frac{u_{t}}{p_{t}} \text {. }
$$

Н е о $б$ х о д и м о с т ь. По лемме $4 \mathrm{z} \in \Omega(J)$. По критерию оптимальности в задаче выпуклого программирования с линейными ограничениями (вариант теоремы Куна-Таккера, см., например, теорему 3.5.4 из [11, гл. 3]) точка $\mathbf{z}$ удовлетворяет системе

$$
\begin{gathered}
\ln \frac{z_{t}}{p_{t}}+1=\sum_{(a, b) \in A \times B} \sigma_{a, b} \ln \frac{a_{t}}{b_{t}}+\beta, \quad t \in J, \\
\sum_{t \in J} z_{t} \ln \frac{a_{t}}{b_{t}} \geqslant 0 \quad \text { для всех }(\mathbf{a}, \mathbf{b}) \in A \times B, \\
\sum_{(a, b) \in A \times B} \sigma_{a, b} \sum_{t \in J} z_{t} \ln \frac{a_{t}}{b_{t}}=0
\end{gathered}
$$

при некоторых неотрицательных $\sigma_{a, b},(\mathbf{a}, \mathbf{b}) \in A \times B$ и $\beta \in R$. Отсюда следуют условия (8).

Д о с т а т о ч н о с т ь. Из (8) следует (9) при $\beta=1-\ln \sum_{t \in J} p_{t} \sigma_{t}$. По тому же критерию оптимальности

$$
\min _{u \in M(A, B)} \sum_{t \in J} u_{t} \ln \frac{u_{t}}{p_{t}}=\sum_{t \in J} z_{t} \ln \frac{z_{t}}{p_{t}}=I(\mathbf{z}, \mathbf{p})=-\ln \sum_{t \in J} p_{t} \sigma_{t}
$$


Следуюший пример (полезный при решении задачи об $r$-гранной игральной кости) показывает, что теорема 3 иногда позволяет найти $\rho(\mathbf{p} ; A, B)$ в явном виде.

П р и м е р 1. Пусть $A \cap B=\varnothing$,

$A \cup B \subseteq M_{r}(\varepsilon)=\{(\varepsilon, \delta, \ldots, \delta),(\delta, \varepsilon, \delta, \ldots, \delta), \ldots,(\delta, \delta, \ldots, \delta, \varepsilon)\}$,

$$
\begin{aligned}
\left|M_{r}(\varepsilon)\right| & =r, \quad \varepsilon \in\left(\frac{1}{r}, 1\right), \quad \delta=\frac{1-\varepsilon}{r-1}, \\
|A| & =k, \quad \mathbf{p}=\left(p_{1}, \ldots, p_{r}\right) \in B, \quad p_{j}=\varepsilon, \\
J_{A}=\left\{i: a_{i}\right. & \left.=\varepsilon \text { при некотором } \mathbf{a}=\left(a_{1}, \ldots, a_{r}\right) \in A\right\}, \\
J_{B}=\left\{i: b_{i}\right. & \left.=\varepsilon \text { при некотором } \mathbf{b}=\left(b_{1}, \ldots, b_{r}\right) \in B\right\} .
\end{aligned}
$$

Тогда условия теоремы 3 выполняются для $M(A, B)$ при $J=\{1, \ldots, r\}$.

Действительно, пусть

$$
\begin{gathered}
\frac{|A|}{|A|+|B|}<u<1, \quad v>0, \\
v(r-|A|-|B|)<1-u, \quad u_{i}=\frac{u}{|A|} \quad \text { для } i \in J_{A}, \\
u_{i}=\frac{1}{|B|}(1-u-v(r-|A|-|B|)) \quad \text { для } i \in J_{B},
\end{gathered}
$$

$u_{i}=v$ для остальных $i$. Тогда точка $\left(u_{1}, \ldots, u_{r}\right) \in M_{+}(A, B) \cap \Omega_{+}$. Следовательно, $s(M(A, B))=r, J=J(M(A, B))=\{1, \ldots, r\}$. Нетрудно проверить, что условия (8) выполняются при следующих числах $\sigma_{a, b}: \sigma_{a, b}=0$, если $a \in A, b \in B \backslash\{\mathbf{p}\}$ и $\sigma_{a, p}=\sigma=1 /(k+1)$, если $a \in A$. При этом $\sigma_{i}=(\varepsilon / \delta)^{\sigma}$, если $i \in J_{A}, \sigma_{j}=(\varepsilon / \delta)^{\sigma-1}, \sigma_{i}=1$ для остальных $r-k-1$ значений $i$. Тогда

$$
\rho(\mathbf{p} ; A, B)=-\ln \sum_{t \in J} p_{t} \sigma_{t}=\rho_{k}^{*},
$$

где $\rho_{k}^{*}$ определено в (3).

Теорема 4. Пусть $A, B, H$ - непустые конечные подмножества множества $\Omega_{+}, M(A, H), M(B, H)$ - непустые множества, $\mathbf{p} \in \Omega_{+} \backslash$ $M(B, H)$ и $\rho(\mathbf{p} ; A, H) \leqslant \rho(\mathbf{p} ; B, H)$. Тогда при некотором $\mathbf{b} \in B$

$$
M(A, b) \neq \varnothing, \quad \rho(\mathbf{p} ; A, \mathbf{b}) \leqslant \rho(\mathbf{p} ; A, H) .
$$

Док аз а т е ль с т в о. Положим $D=M(A, H) \cap M_{+}(B, H), C=$ $M(A, H) \backslash M_{+}(B, H)$. Пусть $\rho(\mathbf{p} ; A, H)=I(\mathbf{w}, \mathbf{p})$ при некотором $\mathbf{w} \in$ $M(A, H)$. Если w $\in D$, то $(1-\varepsilon) \mathbf{w}+\varepsilon \mathbf{p} \in M_{+}(B, H)$ при достаточно малом $\varepsilon \in(0 ; 1)$. По лемме 4 точка $\mathbf{w}$ не является точкой минимума $I(\mathbf{u}, \mathbf{p})$ на $M(B, H)$,

$$
\rho(\mathbf{p} ; A, H)=I(\mathbf{w}, \mathbf{p})>\min _{u \in M(B, H)} I(\mathbf{u}, \mathbf{p})=\rho(\mathbf{p} ; B, H),
$$


что противоречит условию теоремы. Следовательно,

$$
\mathbf{w} \in C, \quad \mathbf{w} \in M(A, H) \cap M(\mathbf{h}, \mathbf{b})
$$

при некоторых $\mathbf{h} \in H, \mathbf{b} \in B$. Тогда $\mathbf{w} \in M(A, \mathbf{b})$,

$$
\rho(\mathbf{p} ; A, H)=I(\mathbf{w}, \mathbf{p}) \geqslant \min _{u \in M(A, b)} I(\mathbf{u}, \mathbf{p})=\rho(\mathbf{p} ; A, \mathbf{b}) .
$$

Следствие 1. Пусть $A, H$ - непустые конечные подмножества множества $\Omega_{+}, \mathbf{b} \in \Omega_{+}, M(A, H) \neq \varnothing, \mathbf{p} \in \Omega_{+} \backslash M(\mathbf{b}, H) u \rho(\mathbf{p} ; A, H) \leqslant$ $\rho(\mathbf{p} ; b, H)$. Toгдa $\rho(\mathbf{p} ; A, b) \leqslant \rho(\mathbf{p} ; A, H)$.

Следствие 2. Пусть $\mathbf{p}, \mathbf{a}, \mathbf{b}-$ три различных элемента множества $\Omega_{+} u \rho(\mathbf{p}, \mathbf{a}) \leqslant \rho(\mathbf{p}, \mathbf{b})$. Tогда $\rho(\mathbf{p} ; \mathbf{a}, \mathbf{b}) \leqslant \rho(\mathbf{p}, \mathbf{a})$.

\section{3. Вычисление расстояния $\rho(\mathbf{p} ; \mathbf{a}, \mathbf{b})$}

Применение теоремы 3 при одноэлементных $A=\{\mathbf{a}\}, B=\{\mathbf{b}\}$ и при $\mathbf{p} \in \Omega_{+} \backslash M(\mathbf{a}, \mathbf{b})$ дает следующие равенства

$$
\rho(\mathbf{p} ; \mathbf{a}, \mathbf{b})=I(\mathbf{z}, \mathbf{p})=-\ln \min _{x \geqslant 0} f(x ; \mathbf{p}, \mathbf{a}, \mathbf{b})=-\ln f\left(\sigma_{i} ; \mathbf{p}, \mathbf{a}, \mathbf{b}\right)
$$

где $f(x ; \mathbf{p}, \mathbf{a}, \mathbf{b})=\sum_{k=1}^{r} p_{k}\left(a_{k} / b_{k}\right)^{x}, \sigma>0$ - ецинственный вещественный корень уравнения $f^{\prime}(x ; \mathbf{p}, \mathbf{a}, \mathbf{b})=0, \mathbf{z}=\left(z_{1}, \ldots, z_{r}\right), z_{k}=p_{k}\left(a_{k} / b_{k}\right)^{\sigma} \times$ $(f(\sigma ; \mathbf{p}, \mathbf{a}, \mathbf{b}))^{-1}$ для $k=1, \ldots, r$.

Теорема 5. Пусть $\mathbf{a} \in \Omega_{+}, \mathbf{b} \in \Omega_{+}, \mathbf{a} \neq \mathbf{b}, \mathbf{p} \in \Omega_{+} \backslash M(\mathbf{a}, \mathbf{b}), \mathbf{p}^{*}=$ $\left(p_{1}^{*}, \ldots, p_{\tau}^{*}\right)$, где $p_{k}^{*}=p_{k}\left(a_{k} / b_{k}\right)^{\tau}$ для $k=1, \ldots, r, \tau-$ единственньй на $(\sigma, \infty)$ корень уравнения $f(x ; \mathbf{p}, \mathbf{a}, \mathbf{b})=1$. Тогда

$$
\begin{aligned}
& \rho(\mathbf{p} ; \mathbf{a}, \mathbf{b})=\rho\left(\mathbf{p}^{*} ; \mathbf{b}, \mathbf{a}\right) \\
& \rho(\mathbf{p} ; \mathbf{a}, \mathbf{b})=\min _{u \in \Omega} \max \left(I(\mathbf{u}, \mathbf{p}), I\left(\mathbf{u}, \mathbf{p}^{*}\right)\right)
\end{aligned}
$$

Д о к а з а т е л ь с т в о. Из (10) следует, что $\rho\left(\mathbf{p}^{*} ; \mathbf{b}, \mathbf{a}\right)=I\left(\mathbf{z}^{*}, \mathbf{p}^{*}\right)$, где $\mathbf{z}^{*}=\left(z_{1}^{*}, \ldots, z_{r}^{*}\right), z_{k}^{*}=p_{k}^{*}\left(b_{k} / a_{k}\right)^{\sigma^{*}}\left(f\left(\sigma^{*} ; \mathbf{p}^{*}, \mathbf{b}, \mathbf{a}\right)\right)^{-1}$ для $k=1, \ldots, r$, $\sigma^{*}>0$ единственный вещественный корень уравнения $f^{\prime}\left(x ; p^{*}\right.$, $\mathbf{b}, \mathbf{a})=0$. Это уравнение равносильно уравнению $f^{\prime}(\tau-x ; \mathbf{p}, \mathbf{a}, \mathbf{b})=0$. Следовательно, $\sigma^{*}=\tau-\sigma, \mathbf{z}^{*}=\mathbf{z}, I\left(\mathbf{z}^{*}, \mathbf{p}^{*}\right)=I(\mathbf{z}, \mathbf{p})$, что доказываeт (11).

Пусть

$$
\begin{aligned}
& \Omega_{1}=\left\{\mathbf{u}: \mathbf{u} \in \Omega, I(\mathbf{u}, \mathbf{p}) \geqslant I\left(\mathbf{u}, \mathbf{p}^{*}\right)\right\}=M(\mathbf{a}, \mathbf{b}) \\
& \Omega_{2}=\left\{\mathbf{u}: \mathbf{u} \in \Omega, I(\mathbf{u}, \mathbf{p}) \leqslant I\left(\mathbf{u}, \mathbf{p}^{*}\right)\right\}=M(\mathbf{b}, \mathbf{a})
\end{aligned}
$$


Тогда

$$
\begin{aligned}
& \min _{u \in \Omega} \max \left(I(\mathbf{u}, \mathbf{p}), I\left(\mathbf{u}, \mathbf{p}^{*}\right)\right) \\
& \quad=\min \left(\min _{u \in \Omega_{1}} \max \left(I(\mathbf{u}, \mathbf{p}), I\left(\mathbf{u}, \mathbf{p}^{*}\right)\right), \min _{u \in \Omega_{2}} \max \left(I(\mathbf{u}, \mathbf{p}), I\left(\mathbf{u}, \mathbf{p}^{*}\right)\right)\right) \\
& \quad=\min \left(\min _{u \in M(a, b)} I(\mathbf{u}, \mathbf{p}), \min _{u \in M(b, a)} I\left(\mathbf{u}, \mathbf{p}^{*}\right)\right)=\rho(\mathbf{p} ; \mathbf{a}, \mathbf{b}),
\end{aligned}
$$

что доказывает (12).

3 а м е ч а н й е 2. Полагая $c_{k}=a_{k} / b_{k}, k=1, \ldots, r$, нетрудно доказать следуюшие оценки полезные при вычислении $\sigma, \tau$ как корней указанных выше уравнений:

$$
\begin{aligned}
& \sigma<S=-\left(\sum_{k=1}^{r} p_{k} \ln c_{k}\right)\left(\sum_{k=1}^{r} \chi\left(c_{k}>1\right) p_{k}\left(\ln c_{k}\right)^{2}\right)^{-1} \\
& \tau<T=\left(e \sum_{k=1}^{r} \chi\left(c_{k}>1\right) p_{k} \ln c_{k}\right)^{-1}
\end{aligned}
$$

3 а м е ч а н и е 3. Пусть $\mathbf{b}=\mathbf{p} \neq \mathbf{a}$. Тогда $\mathbf{p}^{*}=\mathbf{a}, 0<\sigma<1$, $\tau=1$, из равенств $(10),(11)$ следует, что

$$
\rho(\mathbf{p} ; \mathbf{a}, \mathbf{p})=\rho(\mathbf{p} ; \mathbf{p}, \mathbf{a})=\rho(\mathbf{p}, \mathbf{a})=\rho(\mathbf{a}, \mathbf{p}),
$$

где

$$
\rho(\mathbf{p}, \mathbf{a})=-\ln \min _{0 \leqslant x \leqslant 1} \sum_{k=1}^{r} p_{k}\left(\frac{\mathbf{a}_{k}}{\mathbf{p}_{k}}\right)^{x}
$$

-- расстояние Чернова между а и р (см. [2]), а из (11), (12) следуют известные выражения для расстояния Чернова:

$$
\rho(\mathbf{p}, \mathbf{a})=\rho(\mathbf{a}, \mathbf{p})=I(\mathbf{z}, \mathbf{p})=I(\mathbf{z}, \mathbf{a})=\min _{u \in \Omega} \max (I(\mathbf{u}, \mathbf{p}), I(\mathbf{u}, \mathbf{a}))
$$

(см. формулы $(2.20),(2.21)$ в $[7$, гл. $3, \S 2],(4.11)$ в $[1, \S 4],(8.22),(8.23)$ в $[6, \S 8])$.

3 а м е ч а н и е 4 . Если $r=2, \mathbf{p}, \mathbf{a}, \mathbf{b} \in \Omega_{+}, \mathbf{a} \neq \mathbf{b}, \mathbf{p} \notin M(\mathbf{a}, \mathbf{b})$, то

$$
\rho(\mathbf{p} ; \mathbf{a}, \mathbf{b})=-\ln \left(p_{1}\left(\frac{a_{1}}{b_{1}}\right)^{\sigma}+p_{2}\left(\frac{a_{2}}{b_{2}}\right)^{\sigma}\right)
$$

где

$$
\begin{aligned}
\sigma & =\left(\ln \frac{p_{2}}{p_{1}}+\ln \frac{\delta_{2}}{\delta_{1}}\right)\left(\delta_{1}+\delta_{2}\right)^{-1}, \\
\delta_{1} & =\ln \frac{a_{1}}{b_{1}}, \quad \delta_{2}=\ln \frac{b_{2}}{a_{2}}
\end{aligned}
$$


С помоцью (13) можно показать (на примерах), что при любой перестановке $\mathbf{p}^{\prime}, \mathbf{a}^{\prime}, \mathbf{b}^{\prime}$ векторов $\mathbf{p}, \mathbf{a}, \mathbf{b}$, несовпадающей $\mathbf{c} \mathbf{p}, \mathbf{a}, \mathbf{b}$, существуют такие $\mathbf{p}, \mathbf{a}, \mathbf{b}$, что $\rho(\mathbf{p} ; \mathbf{a}, \mathbf{b})>0, \rho\left(\mathbf{p}^{\prime} ; \mathbf{a}^{\prime}, \mathbf{b}^{\prime}\right)=0$. Существуют также такие $\mathbf{p}, \mathbf{a}, \mathbf{b}$, что $0<\rho(\mathbf{p} ; \mathbf{a}, \mathbf{b})<\rho(\mathbf{p}, \mathbf{a})<\rho(\mathbf{p}, \mathbf{b})$. Далее, при $\rho(\mathbf{p}, \mathbf{a})<\rho(\mathbf{p}, \mathbf{b})$ величина $\rho(\mathbf{p} ; \mathbf{b}, \mathbf{a})$ может быть меньше $\rho(\mathbf{p}, \mathbf{a})$, больше $\rho(\mathbf{p}, \mathbf{b})$, находиться между ними, а величины $\rho(\mathbf{p} ; \mathbf{a}, \mathbf{h}), \rho(\mathbf{p} ; \mathbf{b}, \mathbf{h})$ $(\rho(\mathbf{p} ; \mathbf{h}, \mathbf{a}), \rho(\mathbf{p} ; \mathbf{h}, \mathbf{b}))$ могут удовлетворять как неравенствам $0<\rho(\mathbf{p} ;$ $\mathbf{a}, \mathbf{h})<\rho(\mathbf{p} ; \mathbf{b}, \mathbf{h})(0<\rho(\mathbf{p} ; \mathbf{h}, \mathbf{a})<\rho(\mathbf{p} ; \mathbf{h}, \mathbf{b}))$, так и неравенствам $\rho(\mathbf{p} ;$ $\mathbf{a}, \mathbf{h})>\rho(\mathbf{p} ; \mathbf{b}, \mathbf{h})>0(\rho(\mathbf{p} ; \mathbf{h}, \mathbf{a})>\rho(\mathbf{p} ; \mathbf{h}, \mathbf{b})>0)$. Эти примеры дополняют содержание следствия 2 .

\section{4. Приложение к задаче различения нескольких простых гипотез}

Задачу различения нескольких простых гипотез будем решать, используя теорию статистических игр с фиксированным объемом выборки (см., например, [3, гл. 3,6, 7]).

Пусть на конечном пространстве $E=\left\{E_{1}, \ldots, E_{N}\right\}$ элементарных событий определены положительные вероятностные меры $\mu_{1}, \ldots, \mu_{m}$ : $\mu_{i}\left(E_{j}\right)>0$ для $j=1, \ldots, N, i=1, \ldots, m, \sum_{j=1}^{N} \mu_{i}\left(E_{j}\right)=1$ для $i=$ $1, \ldots, m, m \geqslant 2$. Гипотеза $H_{t}, t=1, \ldots, m$, состоит в том, что событие $E_{v}$ появляется с вероятностью $\mu_{t}\left(E_{v}\right)$ при каждом $v=1, \ldots, N$. Рассмотрим решаюшее правило $D(\mathbf{k})$, где $\mathbf{k}=\left(k_{1}, \ldots, k_{N}\right), k_{v} \in\{0,1, \ldots, m\}$ для $v=1, \ldots, N$, которое каждому элементарному событию $E_{v}, v=1, \ldots, N$, ставит в соответствие решение

$$
d\left(E_{v}, \mathbf{k}\right)=d\left(E_{v}\right) \subseteq\left\{H_{1}, \ldots, H_{m}\right\}, \quad\left|d\left(E_{v}\right)\right|=k_{v} .
$$

По этому правилу при появлении события $E_{v}$ статистик принимает множество $d\left(E_{v}\right)$ гипотез, т.е. делает заключение: верна одна из гипотез $H_{t} \in d\left(E_{v}\right)$, иными словами дает ответ об истинной гипотезе в $k_{v}$ вариантах, если $1 \leqslant k_{v} \leqslant m$; при $k_{v}=0$ не принимается ни одна из гипотез $H_{1}, \ldots, H_{m}$. Если $t_{1}, \ldots, t_{s}$ - множество всех различных чисел среди $k_{1}, \ldots, k_{N}$, то задание правила $D(\mathbf{k})$ равносильно разбиению пространства $E$ на $C_{m}^{t_{1}}+\cdots+C_{m}^{t_{s}}$ попарно непересекающихся возможно пустых областей $E(J)=\left\{E_{v}: d\left(E_{v}\right)=\left\{H_{j}, j \in J\right\}\right\}$ принятия множеств $\left\{H_{j}, j \in J\right\}$ гипотез, $J \subseteq\{1, \ldots, m\},|J| \in\left\{t_{1}, \ldots, t_{s}\right\}$. Заметим, что числа $k_{v}$ и множества $d\left(E_{v}\right)$ принимаемых гипотез могут не задаваться заранее, а определяться при появлении события $E_{v}$ с помощью некоторого алгоритма.

Вероятность не найти истинную гипотезу $H_{t}$ в принятом множестве гипотез при решающем правиле $D(\mathbf{k})$ равна

$$
\alpha_{t}=\alpha_{t}(D(\mathbf{k}))=\sum_{v=1}^{N} \mu_{t}\left(E_{v}\right) \chi\left(H_{t} \notin d\left(E_{v}\right)\right) .
$$


3 а м е ч а н и е 5 . Не более $k_{0}=\min _{1 \leqslant i \leqslant N} k_{i}$ величин $\alpha_{1}, \ldots, \alpha_{m}$ равны 0 . (В противном случае $H_{t} \in \cap_{v=1}^{N} d\left(E_{v}\right)$ при более чем $k_{0}$ значениях $t$, т.е. $k_{v}>k_{0}$ при всех $v=1, \ldots, N$.)

3 а м е а н и е 6 . Если два правила $D(\mathbf{k}), D(\mathbf{l})$ таковы, что $\left.d\left(E_{v}, \mathbf{k}\right)\right) \subseteq d\left(E_{v}, \mathbf{l}\right)$ при каждом $v=1, \ldots, N$, то $\alpha_{t}(D(\mathbf{l})) \leqslant \alpha_{t}(D(\mathbf{k}))$ для $t=1, \ldots, m$.

Рассмотрим задачу построения решаюшего правила $D(\mathbf{k})$, которое минимизирует величину $\alpha=\sum_{t=1}^{m} \lambda_{t} \alpha_{t}$ при заданных $\lambda_{t}>0, t=1, \ldots, m$, удовлетворяющих условию $\sum_{t=1}^{m} \lambda_{t}=1\left(\lambda_{t}\right.$ - априорная вероятность гипотезы $H_{t}$ ). Так как

$$
\alpha=\alpha(D(\mathbf{k}))=\sum_{v=1}^{N} \sum_{t=1}^{m} \lambda_{t} \mu_{t}\left(E_{v}\right) \chi\left(H_{t} \notin d\left(E_{v}\right)\right)
$$

то при фиксированном $\mathbf{k}$ решающее правило, минимизирующее величину $\alpha$, можно построить следуюшим образом. При каждом $v \in\{1, \ldots, N\}$ таком, что $k_{v} \in\{1, \ldots, m\}$, рассмотрим одно (любое) из упорядочений $m$ величин $L_{t}\left(E_{v}\right)=\lambda_{t} \mu_{t}\left(E_{v}\right), t=1, \ldots, m$, в порядке невозрастания:

$$
L_{t_{1}}\left(E_{v}\right) \geqslant \cdots \geqslant L_{t_{m}}\left(E_{v}\right)
$$

Положим $d\left(E_{v}\right)=\left\{H_{t_{1}}, \ldots, H_{k_{v}}\right\}$; при $k_{v}=0$ положим $d\left(E_{v}\right)=\varnothing$. При этом минимизируется каждая из $N$ сумм $\sum_{t=1}^{m} L_{t}\left(E_{v}\right) \chi\left(H_{t} \notin d\left(E_{v}\right)\right)$, $v=1, \ldots, N$, и, следовательно, величина $\alpha$. Построенное таким образом при заданных $\lambda=\left(\lambda_{1}, \ldots, \lambda_{m}\right), \mathbf{k}=\left(k_{1}, \ldots, k_{N}\right)$ с помощью неравенств (15) решающее правило назовем байесовским правилом $D_{B}(\lambda, \mathbf{k})$. При $\mathbf{k}=(1, \ldots, 1)$ правила $D(\mathbf{k}), D_{B}(\lambda, \mathbf{k})$ рассматривались, например, в $[12$, гл. $3, \S 1]$.

Пусть $k \in\{1, \ldots, m-1\}$. При каждом $v \in\{1, \ldots, N\}$ найдем с помощью неравенств (15) минимальное $\mathscr{K} \in\{k, k+1, \ldots, m\}$ такое, что $L_{t_{\mathscr{K}}}\left(E_{v}\right)>L_{t_{\mathscr{X}+1}}\left(E_{v}\right)$, полагая при этом $L_{t_{m+1}}\left(E_{v}\right)=0$. Если при каждом $v \in\{1, \ldots, N\}$ принимается решение

$$
d\left(E_{v}\right)=\left\{H_{t_{1}}, \ldots, H_{t_{k}}\right\}\left(d\left(E_{v}\right)=\left\{H_{t_{1}}, \ldots, H_{t_{K}}\right\}\right)
$$

то получающееся при этом байесовское правило назовем байесовским правилом $D_{B}(\lambda, k)$ (строгим байесовским правилом $D_{s B}(\lambda, k)$ ). Правило $D_{B}(\lambda, k)$ - одно из правил $D(\mathbf{k})$, где $\mathbf{k}=(k, \ldots, k)$, правило $D_{s B}(\lambda, k)$ - одно из правил $D(\mathbf{k})$, где $\mathbf{k}=\left(k_{1}, \ldots, k_{N}\right), k_{v} \in\{k, \ldots, m\}$ при $v=1, \ldots, N$.

Пусть $E_{\lambda}(J), E_{s \lambda}(J)$ - области $E(J)$ для правил $D_{B}(\lambda, k), D_{s B}(\lambda, k)$ соответственно, $A(t, k)=\{J:|J|=k, t \notin J\}$ для $k \in\{1, \ldots, m-1\}$, 
$t \in\{1, \ldots, m\},|A(t, k)|=C_{m-1}^{k}$. С учетом замечания 6 из (14) следует оценка:

$$
\begin{aligned}
\alpha_{t}\left(D_{s B}(\lambda, k)\right) & =\sum_{\mathscr{K}=k}^{m-1} \sum_{J \in A(t, \mathscr{K})} \mu_{t}\left(E_{s \lambda}(J)\right) \\
& \leqslant \alpha_{t}\left(D_{B}(\lambda, k)\right)=\sum_{J \in A(t, k)} \mu_{t}\left(E_{\lambda}(J)\right)
\end{aligned}
$$

для $k \in\{1, \ldots, m-1\}, t \in\{1, \ldots, m\}$.

Построим теперь байесовские решающие правила для различения $m$ гипотез $H\left(\mathbf{p}_{j}, n\right), j=1, \ldots, m$. На пространстве $E=\Omega_{n}$ элементарных событий $\underline{\mathbf{x}}$ определим меры $\mu_{j}(\underline{\mathbf{x}})=\mathbf{P}_{n}\left(\underline{\mathbf{x}}, \mathbf{p}_{j}\right), j=1, \ldots, m$. Пусть $J \subseteq$ $\{1, \ldots, m\}, 1 \leqslant|J| \leqslant m-1$. Положим

$$
\begin{aligned}
A_{J} & =\left\{\mathbf{p}_{j}: j \in J\right\}, & & B_{j}=\left\{\mathbf{p}_{j}: j \in\{1, \ldots, m\} \backslash J\right\}, \\
M(J) & =M\left(A_{J}, B_{J}\right), & & M_{+}(J)=M_{+}\left(A_{J}, B_{J}\right) .
\end{aligned}
$$

При $\lambda=\theta=(1 / m, \ldots, 1 / m), k \in\{1, \ldots, m-1\}$ положим

$$
D_{B}(\theta, k)=D_{B}(k)=D_{B}(n ; k), \quad D_{s B}(\theta, k)=D_{s B}(k)=D_{s B}(n ; k) \text {. }
$$

Для правил $D_{B}(n ; k), D_{s B}(n ; k)$ имеем:

$E_{\theta}(J) \subseteq M(J) \cap \Omega_{n}, \quad E_{s \theta}(J)=M_{+}(J) \cap \Omega_{n}, \quad$ если $\quad|J|=k$, $E_{s \theta}(J) \subseteq M_{+}(J) \cap \Omega_{n}, \quad$ если $|J|>k$.

Отсюда и из (16) при любом $t \in\{1, \ldots, m\}$ следуют ощенки

$$
\begin{aligned}
& \alpha_{t}\left(D_{s B}(n ; k)\right) \leqslant \sum_{\mathscr{K}=k}^{m-1} \sum_{J \in A(t, \mathscr{K})} \mathbf{P}_{n}\left(M_{+}(J), \mathbf{p}_{t}\right) \\
& \sum_{J \in A(t, k)} \mathbf{P}_{n}\left(M_{+}(J), \mathbf{p}_{t}\right) \leqslant \alpha_{t}\left(D_{s B}(n ; k)\right) \\
& \leqslant \alpha_{t}\left(D_{B}(n ; k)\right) \leqslant \sum_{J \in A(t, k)} \mathbf{P}_{n}\left(M(J), \mathbf{p}_{t}\right) .
\end{aligned}
$$

Теорема 6. Пусть $t \in\{1, \ldots, m\}, k \in\{1, \ldots, m-1\}$,

$$
\begin{aligned}
B(t, k) & =\{J \in A(t, k): M(J) \neq \varnothing\}, \\
C_{+}(t, k) & =\left\{J \in A(t, k): M_{+}\left(A_{J}, \mathbf{p}_{t}\right) \neq \varnothing\right\}, \\
\rho_{t, k} & =\min _{J \in C_{+}(t, k)} \rho\left(\mathbf{p}_{t} ; A_{J}, \mathbf{p}_{t}\right)>0, \quad \text { ecлu } C_{+}(t, k) \neq \varnothing, \\
\bar{\rho}_{t, k} & =\min _{J \in B(t, k)} \rho\left(\mathbf{p}_{t} ; A_{J}, B_{J}\right), \quad \text { ecлu } B(t, k) \neq \varnothing .
\end{aligned}
$$

Тогда для всех $n \geqslant 1$ 
1) если $B(t, k)=\varnothing$, mo $\alpha_{t}\left(D_{s B}(n ; k)\right)=\alpha_{t}\left(D_{B}(n ; k)\right)=0$;

2) если $B(t, k) \neq \varnothing$, mo

$$
\begin{aligned}
\alpha_{t}\left(D_{s B}(n ; k)\right) & \leqslant \alpha_{t}\left(D_{B}(n ; k)\right) \leqslant \sum_{J \in B(t, k)} \exp \left(-n \rho\left(\mathbf{p}_{t} ; A_{J}, B_{J}\right)\right) \\
& \leqslant|B(t, k)| \exp \left(-n \bar{\rho}_{t, k}\right) ;
\end{aligned}
$$

3) если $C_{+}(t, k)=\varnothing$, mo $\alpha_{t}\left(D_{s B}(n ; k)\right)=0$.

4) $\operatorname{Ecлu} C_{+}(t, k) \neq \varnothing, m o$

$$
B(t, k) \neq \varnothing, \quad \alpha_{t}\left(D_{B}(n ; k)\right) \geqslant \alpha_{t}\left(D_{s B}(n ; k)\right)>0
$$

для всех достаточно больших $n$ и

$$
\begin{aligned}
\lim _{n \rightarrow \infty} n^{-1} \ln \alpha_{t}\left(D_{s B}(n ; k)\right) & =-\rho_{t, k} \leqslant \liminf _{n \rightarrow \infty} n^{-1} \ln \alpha_{t}\left(D_{B}(n ; k)\right) \\
& \leqslant \limsup _{n \rightarrow \infty} n^{-1} \ln \alpha_{t}\left(D_{B}(n ; k)\right) \leqslant-\bar{\rho}_{t, k} .
\end{aligned}
$$

Доказа тель с т в о. Утверждения 1), 2) следуют из (18) и теоремы 2.

Докажем утверждение 3). Пусть

$\mathscr{K} \in\{k, \ldots, m-1\}, \quad J=\left(j_{1}, \ldots, j_{\mathscr{K}}\right) \in A(t, \mathscr{K}), \quad I=\left(j_{1}, \ldots, j_{k}\right)$.

Torда

$$
\begin{aligned}
\mathbf{P}_{n}\left(M_{+}(J), \mathbf{p}_{t}\right) & \leqslant \mathbf{P}_{n}\left(M_{+}\left(A_{I}, \mathbf{p}_{t}\right), \mathbf{p}_{t}\right) \\
\sum_{J \in A(t, k)} \mathbf{P}_{n}\left(M_{+}(J), \mathbf{p}_{t}\right) & \leqslant \sum_{I \in A(t, k)} \mathbf{P}_{n}\left(M_{+}\left(A_{I}, \mathbf{p}_{t}\right), \mathbf{p}_{t}\right),
\end{aligned}
$$

а при $\mathscr{K}>k$ имеем оценки

$$
\begin{aligned}
\sum_{J \in A(t, \mathscr{K})} \mathbf{P}_{n}\left(M_{+}(J), \mathbf{p}_{t}\right) & \leqslant \sum_{I \in A(t, k)} \sum_{j_{k}<j_{k+1}<\cdots<j \mathscr{K} \leqslant m} \mathbf{P}_{n}\left(M_{+}\left(A_{I}, \mathbf{p}_{t}\right), \mathbf{p}_{t}\right) \\
& =\sum_{I \in A(t, k)} C_{m-j_{k}}^{\mathscr{K}-k} \mathbf{P}_{n}\left(M_{+}\left(A_{I}, \mathbf{p}_{t}\right), \mathbf{p}_{t}\right) \\
& \leqslant C_{m-k}^{\mathscr{K}-k} \sum_{I \in A(t, k)} \mathbf{P}_{n}\left(M_{+}\left(A_{I}, \mathbf{p}_{t}\right), \mathbf{p}_{t}\right) .
\end{aligned}
$$

Отсюда и из (17) следует оценка

$$
\alpha_{t}\left(D_{s B}(n ; k)\right) \leqslant\left(2^{m-k}-1\right) \sum_{J \in C_{+}(t, k)} \mathbf{P}_{n}\left(M_{+}\left(A_{J}, \mathbf{p}_{t}\right), \mathbf{p}_{t}\right)
$$

и утверждение 3).

Докажем утверждение 4). Пусть $\mathbf{u}=\left(u_{1}, \ldots, u_{r}\right) \in M_{+}\left(A_{K}, \mathbf{p}_{t}\right) \cap \Omega_{n}$ при некотором $K=\left\{i_{1}, \ldots, i_{k}\right\} \in C_{+}(t, k)$ и $\gamma_{i}=\sum_{j=1}^{r} u_{j} \ln p_{i, j}$ для $i=$ 
$1, \ldots, m$. Предположим, что $\mathbf{u} \in E_{s \theta}(J)$ при некотором $J=\left\{j_{1}, \ldots, j_{\mathcal{K}}\right\}$ таком, что $t \in J$. Если $\mathscr{K}=m$, то $J=\{1, \ldots, m\}, K \subseteq J$. Если $\mathscr{K}<m$, то для $j \notin J$ величина $\gamma_{j}<\gamma_{t}$. Следовательно, всегда

$$
\begin{gathered}
K \subseteq J, \\
\min _{i \in K} \gamma_{i}>\gamma_{t} \geqslant \gamma=\min _{j \in J} \gamma_{j} .
\end{gathered}
$$

Пусть $I=\left\{j \in J: \gamma_{j}=\gamma\right\}$. Из (22) следует, что

$$
I \subseteq J, \quad I \cap K=\varnothing .
$$

Из (21), (23) следует, что

$$
|I|+k=|I|+|K| \leqslant \mathscr{K} .
$$

Из способа построения правила $D_{s B}(n ; k)$ следует неравенство $|I| \geqslant \mathscr{K}-$ $k+1$, что противоречит (24). Таким образом, $t \notin J$ и

$$
M_{+}\left(A_{\mathcal{K}}, \mathbf{p}_{t}\right) \cap \Omega_{n} \subseteq \bigcup_{\mathscr{K}=k}^{m-1} \bigcup_{J \in A(t, \mathcal{K})} E_{s \theta}(J) .
$$

Отсюда и из (16) следует оценка

$$
\max _{J \in C_{+}(t, k)} \mathbf{P}_{n}\left(M_{+}\left(A_{J}, \mathbf{p}_{t}\right), \mathbf{p}_{t}\right) \leqslant \alpha_{t}\left(D_{s B}(n ; k)\right) .
$$

Из (25) и теоремы 1 следует, что $\alpha_{t}\left(D_{s B}(n ; k)\right)>0$ при всех достаточно больших $n$, а это с учетом утверждения 1$)$ приводит к неравенству $B(t, k) \neq \varnothing$. Из (20), (25), оценок утверждения 2) с помошью теоремы 1 и утверждения г) следует (19).

Следствие 3. Если $C_{+}(t, k) \neq \varnothing$ и $F(t, k)=B(t, k) \backslash C_{+}(t, k)=\varnothing$, $m o \bar{\rho}_{t, k}=\rho_{t, k} u$

$$
\lim _{n \rightarrow \infty} n^{-1} \ln \alpha_{t}\left(D_{s B}(n ; k)\right)=\lim _{n \rightarrow \infty} n^{-1} \ln \alpha_{t}\left(D_{B}(n ; k)\right)=-\rho_{t, k} .
$$

Д ок аз а т е л с с т в о. Имеем

$$
\begin{aligned}
& \bar{\rho}_{t, k}=\rho^{\prime}=\min _{J \in B(t, k) \cap C_{+}(t, k)} \rho\left(\mathbf{p}_{t} ; A_{J}, B_{J}\right), \quad \text { если } F(t, k)=\varnothing, \\
& \bar{\rho}_{t, k}=\min _{J \in F(t, k)} \rho\left(\mathbf{p}_{t} ; A_{J}, B_{J}\right), \quad \text { если } B(t, k) \cap C_{+}(t, k)=\varnothing, \\
& \bar{\rho}_{t, k}=\min \left(\rho^{\prime}, \min _{J \in F(t, k)} \rho\left(\mathbf{p}_{t} ; A_{J}, B_{J}\right)\right), \quad \text { если } F(t, k) \neq \varnothing \quad \text { и } \\
& \quad B(t, k) \cap C_{+}(t, k) \neq \varnothing .
\end{aligned}
$$

Так как

$$
\rho^{\prime} \geqslant \min _{J \in C_{+}(t, k)} \rho\left(\mathbf{p}_{t} ; A_{J}, B_{J}\right) \geqslant \min _{J \in C_{+}(t, k)} \rho\left(\mathbf{p}_{t} ; A_{J}, \mathbf{p}_{t}\right)=\rho_{t, k} \geqslant \bar{\rho}_{t, k}
$$


(см. (19)), то

$$
\begin{aligned}
& \bar{\rho}_{t, k}=\min \left(\rho_{t, k}, \min _{J \in F(t, k)} \rho\left(\mathbf{p}_{t} ; A_{J}, B_{J}\right)\right), \quad \text { если } F(t, k) \neq \varnothing, \\
& \bar{\rho}_{t, k}=\rho_{t, k}, \quad \text { если } F(t, k)=\varnothing .
\end{aligned}
$$

3 а м е ч а н и е 7. В дополнение к множествам, введенным в теореме 6, положим $C(t, k)=\left\{J \in A(t, k): M\left(A_{J}, \mathbf{p}_{t}\right) \neq \varnothing\right\}, B_{+}(t, k)=\{J \in$ $\left.A(t, k): M_{+}(J) \neq \varnothing\right\}$. Так как $B_{+}(t, k) \subseteq C_{+}(t, k), B(t, k) \subseteq C(t, k)$, то $F(t, k)=\varnothing$, если либо $B_{+}(t, k)=B(t, k)$, либо $C_{+}(t, k)=C(t, k)$.

Так как $\mathbf{p}_{j} \in M_{+}\left(\mathbf{p}_{j}, \mathbf{p}_{t}\right)$ при $j \neq t$, то

$$
C_{+}(t, 1)=C(t, 1)=A(t, 1) .
$$

Условие $C_{+}(t, k)=C(t, k)$ при $k>1$ выполняется, если верно следуюшее утверждение, которое не удалось доказать или опровергнуть. Если $k \geqslant 2, \mathbf{p}_{i}=\left(p_{i, 1}, \ldots, p_{i, r}\right), i=1, \ldots, k, \mathbf{p}=\left(p_{1}, \ldots, p_{r}\right)$ - различные элементы из $\Omega_{+}$и $M\left(\left\{\mathbf{p}_{1}, \ldots, \mathbf{p}_{k}\right\}, \mathbf{p}\right) \neq \varnothing$, то $M_{+}\left(\left\{\mathbf{p}_{1}, \ldots, \mathbf{p}_{k}\right\}, \mathbf{p}\right) \neq \varnothing$.

Укажем простое достаточное условие неравенства

$$
M_{+}\left(\left\{\mathbf{p}_{1}, \ldots, \mathbf{p}_{k}\right\}, \mathbf{p}\right) \neq \varnothing: \min _{1 \leqslant i \leqslant k} \prod_{j \in J}\left(\frac{p_{i, j}}{p_{j}}\right)>1
$$

при некотором $J \subseteq\{1, \ldots, r\}$ (при этом условии точка $\left(v_{1}, \ldots, v_{\tau}\right)$, в которой $v_{j}=1 /|J|$ для $j \in J, v_{j}=0$ для $j \notin J$, принадлежит множеству $\left.M_{+}\left(\left\{\mathbf{p}_{1}, \ldots, \mathbf{p}_{k}\right\}, \mathbf{p}\right)\right)$.

Теория линейных неравенств [10] дает возможность формулировать необхоцимые и достаточные условия для неравенств $M(A, B) \neq \varnothing$, $M_{+}(A, B) \neq \varnothing$. В частности, применяя к системе

$$
\sum_{t=1}^{r} x_{t} \ln \frac{p_{t}}{p_{i, t}} \leqslant 0, \quad i=1, \ldots, k,
$$

теорему 1.9 и дополнение 1 (дополнение 2 ) к ней из $[10$, гл. $1, \S 6]$, легко сформулировать необходимые и достаточные условия для неравенства $M\left(\left\{\mathbf{p}_{1}, \ldots, \mathbf{p}_{k}\right\}, \mathbf{p}\right) \neq \varnothing$ (для неравенства $M\left(\left\{\mathbf{p}_{1}, \ldots, \mathbf{p}_{k}\right\}, \mathbf{p}\right) \cap$ $\Omega_{+} \neq \varnothing$ и, следовательно, для неравенства $\left.M_{+}\left(\left\{\mathbf{p}_{1}, \ldots, \mathbf{p}_{k}\right\}, \mathbf{p}\right) \neq \varnothing\right)$. (При этом используется равносильность неравенств $M_{+}\left(\left\{\mathbf{p}_{1}, \ldots, \mathbf{p}_{k}\right\}\right.$, p) $\neq \varnothing, M\left(\left\{\mathbf{p}_{1}, \ldots, \mathbf{p}_{k}\right\}, \mathbf{p}\right) \cap \Omega_{+} \neq \varnothing$, следуюшая из утверждения б) и из того, что точка $(1+\varepsilon) \mathbf{v}-\varepsilon \mathbf{p} \in M_{+}\left(\left\{\mathbf{p}_{1}, \ldots, \mathbf{p}_{k}\right\}, \mathbf{p}\right) \cap \Omega_{+}$при малом положительном $\varepsilon$ и $\left.\mathbf{v} \in M\left(\left\{\mathbf{p}_{1}, \ldots, \mathbf{p}_{k}\right\}, \mathbf{p}\right) \cap \Omega_{+}\right)$. Необходимые и достаточные условия для неравенства $\left.M_{+}\left(\left\{\mathbf{p}_{1}, \ldots, \mathbf{p}_{k}\right\}, \mathbf{p}\right)\right) \neq \varnothing$ можно также сформулировать, применяя теорему 1.12 из $[10$, гл. $1, \S 7]$ к системе

$$
\sum_{t=1}^{r} x_{t} \ln \frac{p_{t}}{p_{i, t}} \leqslant 0, \quad i=1, \ldots, k,-x_{t} \leqslant 0, t=1, \ldots, r .
$$


Покажем, что при $k>1$ множество $C(t, k)$ может быть пустым.

П р и м е р 2. Пусть $m=3, \mathbf{p}_{1}=\left(p_{1}, \ldots, p_{r}\right) \neq\left(q_{1}, \ldots, q_{r}\right)=\mathbf{p}_{2}$, $\mathbf{p}_{3}=\left(a_{1}, \ldots, a_{r}\right)$, где $a_{i}=c^{-1} \sqrt{p_{i} q_{i}}$ для $i=1, \ldots, r, c=\sum_{i=1}^{r} \sqrt{p_{i} q_{i}}<1$ (неравенство Коши здесь строгое, так как не совпадающие $\mathbf{p}_{1}, \mathbf{p}_{2}$ из $\Omega_{+}$ неколлинеарны). Если $C(3,2) \neq \varnothing$, то $M\left(\left\{\mathbf{p}_{1}, \mathbf{p}_{2}\right\}, \mathbf{p}_{3}\right) \neq \varnothing$. Тогда при некотором $\left(u_{1}, \ldots, u_{r}\right) \in \Omega$

$$
\sum_{i=1}^{r} u_{i} \ln \sqrt{\frac{p_{i}}{q_{i}}}+\ln c \geqslant 0, \quad \sum_{i=1}^{r} u_{i} \ln \sqrt{\frac{q_{i}}{p_{i}}}+\ln c \geqslant 0
$$

а это приводит к противоречию: $c \geqslant 1$. Следовательно, $C(3,2)=\varnothing$.

Пусть $T_{k}=\left\{t \in\{1, \ldots, m\}: C_{+}(t, k) \neq \varnothing\right\}, S_{k}=\{t \in\{1, \ldots, m\}:$ $B(t, k) \neq \varnothing\}$ для $k \in\{1, \ldots, m-1\}$. Из утверждений 3$), 4)$ теоремы 6 и замечания 5 следует, что $T_{k} \subseteq S_{k},\left|T_{k}\right| \geqslant m-k$.

Теорема 7. IIусть

$$
k \in\{1, \ldots, m-1\}, \quad \mathbf{k}=(k, \ldots, k), \quad \rho_{k}=\min _{t \in T_{k}} \rho_{t, k}, \quad \bar{\rho}_{k}=\min _{t \in S_{k}} \bar{\rho}_{t, k}
$$

Tогдa

$$
\begin{aligned}
& \lim _{n \rightarrow \infty} n^{-1} \ln \max _{1 \leqslant t \leqslant m} \alpha_{t}\left(D_{s B}(n ; k)\right)=-\rho_{k} \\
& \leqslant \liminf _{n \rightarrow \infty} n^{-1} \ln \max _{1 \leqslant t \leqslant m} \alpha_{t}\left(D_{B}(n ; k)\right) \\
& \leqslant \limsup _{n \rightarrow \infty} n^{-1} \ln \max _{1 \leqslant t \leqslant m} \alpha_{t}\left(D_{B}(n ; k)\right) \leqslant-\bar{\rho}_{k}
\end{aligned}
$$

и для пюбой последовательности правил $D(\mathbf{k})=D(n ; \mathbf{k}), n=1,2, \ldots$, верно неравенство $\liminf \operatorname{si\infty }_{n \rightarrow \infty} n^{-1} \ln \max _{1 \leqslant t \leqslant m} \alpha_{t}(D(n ; k)) \geqslant-\rho_{k}$.

Д о к а з а т е л ь с т в о. Неравенства (27) следуют из утверждений 1), 2), 3), 4) теоремы 6 и утверждения г) п. 1. Предположим, что при некотором $\varepsilon>0$

$$
\liminf _{n \rightarrow \infty} n^{-1} \ln \max _{1 \leqslant t \leqslant m} \alpha_{t}(D(n ; \mathbf{k})) \leqslant-\rho_{k}-\varepsilon .
$$

Тогда для некоторой возрастающей до $\infty$ последовательности натуральных чисел $n_{1}, n_{2}, \ldots$ имеем при достаточно большом $i$ :

$$
\begin{aligned}
& m^{-1} \sum_{t=1}^{m} \alpha_{t}\left(D\left(n_{i} ; \mathbf{k}\right)\right) \leqslant \max _{1 \leqslant t \leqslant m} \alpha_{t}\left(D\left(n_{i} ; \mathbf{k}\right)\right) \\
& \quad \leqslant \exp \left(-n_{i}\left(\rho_{k}+2^{-1} \varepsilon\right)\right)<m^{-1} \max _{1 \leqslant t \leqslant m} \alpha_{t}\left(D_{s B}\left(n_{i} ; k\right)\right) \\
& \quad \leqslant m^{-1} \max _{1 \leqslant t \leqslant m} \alpha_{t}\left(D_{B}\left(n_{i} ; k\right)\right) \leqslant m^{-1} \sum_{t=1}^{m} \alpha_{t}\left(D_{B}\left(n_{i} ; k\right)\right) .
\end{aligned}
$$


Итак,

$$
m^{-1} \sum_{t=1}^{m} \alpha_{t}\left(D\left(n_{i} ; \mathbf{k}\right)\right)<m^{-1} \sum_{t=1}^{m} \alpha_{t}\left(D_{B}\left(n_{i} ; k\right)\right)
$$

что противоречит свойству байесовского правила $D_{B}(n ; k)$ минимизировать величину $m^{-1} \sum_{t=1}^{m} \alpha_{t}\left(D\left(n_{i} ; \mathbf{k}\right)\right)$.

3 а м е ч а н и е 8 . Если $\bar{\rho}_{k}=\rho_{k}, \mathbf{k}=(k, \ldots, k)$, то последовательность правил $D_{B}(n ; k), n=1,2, \ldots$, оптимальна в том смысле, что

$$
\liminf _{n \rightarrow \infty} n^{-1} \ln \max _{1 \leqslant t \leqslant m} \alpha_{t}(D(n ; k)) \geqslant \lim _{n \rightarrow \infty} n^{-1} \ln \max _{1 \leqslant t \leqslant m} \alpha_{t}\left(D_{B}(n ; k)\right)=-\rho_{k} .
$$

Равенство $\bar{\rho}_{k}=\rho_{k}$ верно, если $B(t, k) \subseteq C_{+}(t, k)$ при всех $t \in T_{k}$, так как в этом случае $T_{k}=S_{k}$ и $\bar{\rho}_{t, k}=\rho_{t, k}$ для всех $t \in T_{k}$ (см. следствие 3 и замечание 7 ). Следствием (26) и замечания 7 является утверждение из [5]: последовательность правил $D_{B}(n ; 1), n=1,2, \ldots$, оптимальна в указанном смысле, при этом $\bar{\rho}_{1}=\rho_{1}=\min _{1 \leqslant i<j \leqslant m} \rho\left(\mathbf{p}_{i}, \mathbf{p}_{j}\right)$.

Из (18) можно получить менее точные (но легче вычисляемые) верхние ощенки для $\alpha_{t}\left(D_{B}(n ; k)\right)$, чем оценка в утвержцении 2) теоремы 6. Для этого достаточно заменить в (18) $M(J)=M\left(A_{J}, B_{J}\right)$ любым $M\left(A_{J}^{\prime}, B_{J}^{\prime}\right)$, где $A_{J}^{\prime} \subseteq J, B_{J}^{\prime} \subseteq B_{J}$ и затем применить теорему $2 . \mathrm{B}$ частности, при $B_{J}^{\prime}=\left\{\mathbf{p}_{t}\right\}$ получим оценку

$$
\alpha_{t}\left(D_{B}(n ; k)\right) \leqslant \sum_{J \in B(t, k)} \exp \left(-n \rho\left(\mathbf{p}_{t} ; A_{J}, \mathbf{p}_{t}\right)\right) .
$$

Рассмотрим достаточно просто вычисляемую оценку такого вида:

$$
\alpha_{t}\left(D_{B}(n ; k)\right) \leqslant \sum_{I \in A(t, k)} \exp \left(-n \rho\left(\mathbf{p}_{t} ; \mathbf{p}_{\lambda(I)}, \mathbf{p}_{\mu(I)}\right)\right)
$$

где $\lambda(I) \in I, \mu(I) \in\{1, \ldots, m\} \backslash I$ (при каждом $I$ пару индексов $\lambda(I), \mu(I)$ можно выбрать $k(m-k)$ способами). Задача об оптимальном выборе обобщенных расстояний Чернова в оценке (28) решается с помощью следуюшей теоремы.

Теорема 8. Для $t \in\{1, \ldots, m\}, k \in\{1, \ldots, m-1\}$ лучшей из оченок (28) является оченка

$$
\alpha_{t}\left(D_{B}(n ; k)\right) \leqslant \sum_{i=k}^{m-1} C_{i-1}^{k-1} \exp \left(-n \rho\left(\mathbf{p}_{t}, \mathbf{p}_{s_{i}}\right)\right)
$$

где числа $s_{i}, i=k, \ldots, m-1$, определяются упорядочиванием $m-1$ расстояний Чернова между $\mathbf{p}_{t} u \mathbf{p}_{j}, j \in\{1, \ldots, m\} \backslash\{t\}$ в неубывающем порядке:

$$
\rho\left(\mathbf{p}_{t}, \mathbf{p}_{s_{1}}\right) \leqslant \cdots \leqslant \rho\left(\mathbf{p}_{t}, \mathbf{p}_{s_{m-1}}\right)
$$


Д о к а з ате л ь с т о. Заметим, что

$$
A(t, k)=\bigcup_{j=1}^{m-k} B_{j}
$$

где $B_{j}=\left\{\left(i_{1}, \ldots, i_{k}\right): 1 \leqslant i_{1}<\cdots<i_{k} \leqslant m\right.$, одно из чисел $i_{1}, \ldots, i_{k}$ равно $s_{m-j}$, остальные принадлежат множеству $\left.\left\{s_{1}, \ldots, s_{m-j-1}\right\}\right\},\left|B_{j}\right|=$ $C_{m-1-j}^{k-1}$ для $j=1, \ldots, m-k$; множества $B_{1}, \ldots, B_{m-k}$ попарно не пересекаются. Разобьем сумму в (28) на $m-k$ сумм по множествам $B_{1}, \ldots, B_{m-k}$. С учетом следствия 2 для любого $I=\left(i_{1}, \ldots, i_{k}\right) \in B_{j}$ имеем

$$
\rho\left(\mathbf{p}_{t} ; \mathbf{p}_{\lambda(I)}, \mathbf{p}_{\mu(I)}\right) \leqslant \rho\left(\mathbf{p}_{t} ; \mathbf{p}_{\lambda(I)}\right) \leqslant \rho\left(\mathbf{p}_{t}, \mathbf{p}_{\mathbf{s}_{m-j}}\right) .
$$

Полагая же $\lambda(I)=s_{m-j}, \mu(I)=t$, получим

$$
\rho\left(\mathbf{p}_{t} ; \mathbf{p}_{\lambda(I)}, \mathbf{p}_{\mu(I)}\right)=\rho\left(\mathbf{p}_{t}, \mathbf{p}_{s_{m-j}}\right) \text {. }
$$

Следовательно, оптимальная оценка (28) такова:

$$
\begin{aligned}
& \alpha_{t}\left(\left(D_{B}(n ; k)\right)\right) \leqslant \sum_{j=1}^{m-k} \sum_{I \in B_{j}} \exp \left(-n \rho\left(\mathbf{p}_{t}, \mathbf{p}_{s_{m-j}}\right)\right) \\
& =\sum_{j=1}^{m-k} C_{m-1-j}^{k-1} \exp \left(-n \rho\left(\mathbf{p}_{t}, \mathbf{p}_{s_{m-j}}\right)\right)=\sum_{i=k}^{m-1} C_{i-1}^{k-1} \exp \left(-n \rho\left(\mathbf{p}_{t}, \mathbf{p}_{s_{i}}\right)\right) .
\end{aligned}
$$

П р и м е р 3. (Задача об $r$-гранной игральной кости). В [3, гл. 6, $\S 4]$ для различения гипотез $H\left(\mathbf{p}_{i}, n\right), i=1, \ldots, r$, где $\left\{\mathbf{p}_{1}, \ldots, \mathbf{p}_{r}\right\}=$ $M_{r}(\varepsilon), M_{r}(\varepsilon)$ определено в примере 1 , построено правило $D_{B}(n ; 1)$. Пусть надо различить гипотезы $H\left(\mathbf{p}_{i}, n\right), i \in J, J \subseteq\{1, \ldots, r\},|J|=m \geqslant 2$, по частотам $x_{1}, \ldots, x_{r}$ исходов. Упорядочим частоты $x_{j}, j \in J: x_{t_{1}} \geqslant$ $\cdots \geqslant x_{t_{m}}\left(t_{1}, \ldots, t_{m}\right.$ - перестановка элементов множества $\left.J\right)$. Тогда при $k \in\{1, \ldots, m-1\}$ по правилу $D_{B}(n ; k)$ принимается множество $\left\{H_{t_{1}}, \ldots, H_{t_{k}}\right\}$, а по правилу $D_{s B}(n ; k)$ - множество $\left\{H_{t_{1}}, \ldots, H_{t_{\mathscr{K}}}\right\}$, где $\mathscr{K}=\min \left\{i: i \in\{k, \ldots, m\}, x_{t_{i}}>x_{t_{i+1}}\right\}$ и $x_{t_{m+1}}<0$. С помошью теоремы 6 и с учетом примера 1 получаются оценки:

$$
\begin{aligned}
\alpha_{t}\left(D_{B}(n ; k)\right) & \leqslant C_{m-1}^{k} \exp \left(-n \rho_{k}^{*}\right) \\
\lim _{n \rightarrow \infty} n^{-1} \ln \alpha_{t}\left(D_{s B}(n ; k)\right) & =\lim _{n \rightarrow \infty} n^{-1} \ln \alpha_{t}\left(D_{B}(n ; k)\right)=-\rho_{k}^{*} .
\end{aligned}
$$

Из теоремы 8 следует ощенка

$$
\alpha_{t}\left(D_{B}(n ; k)\right) \leqslant C_{m-1}^{k} \exp \left(-n \rho_{1}^{*}\right) .
$$

Автор благодарен А. М. Зубкову за замечания и указания, которые помогли улучшить стиль текста и доказать лемму 3 и теорему 2 для более широких классов множеств $\omega$ (автор сначала доказал лемму 3 для 
$\omega=M_{+}(A, B)$, а теорему 2 для $\omega$ из утверждения в) при дополнительном условии $\left.\max _{1 \leqslant i \leqslant t} \sup _{u \in \Omega_{+}}\left\|\operatorname{grad} g_{i}(\mathbf{u})\right\|<\infty\right)$.

\section{СПИСОК ЛИТЕРАТУРЫ}

1. Морозова $E$. A., Ченцов $H$. $H$. Естественная геометрия семейств вероятностных законов. - Итоги науки и техники. Современные проблемы математики. Фундаментальные направления, ВИНИТИ, 1991 т. 83, с. 133-265.

2. Chernoff $H$. A measure of asymptotic efficiency for tests of a hypothesis based on the sum of observations. - Ann. Math. Statist., 1952, v. 23, № 4, p. 493-507.

3. Блекуэлл Д., Гириик М. А. Теория игр и статистических решений. М.: ИЛ, 1958, $374 \mathrm{c}$.

4. Рао C. P. Линейные статистические методы. М.: Наука, 1968, 547 с.

5. Салихов H. П. Асимптотические свойства вероятностей ошибок критериев для различения нескольких мультиномиальных схем испытаний. - Докл. АН СССР, 1973 , т. 209 , № 1 , с. $54-57$.

6. Ченцов $H . H$. Статистические решающие правила и оптимальные выводы. М.: Наука, 1972, $520 \mathrm{c.}$

7. Кульбак C. Теория информации и статистика. М.: Наука, 1967, 408 с.

8. Hoeffding $W$. Asymptotically optimal tests for multinomial distributions. - Ann. Math. Statist., 1965, v. 36, № 2, p. 369-401.

9. Фано Р. Передача информации. Статистическая теория связи. М.: Мир, 1965, $438 \mathrm{c}$.

10. Чержиков С. Н. Линейные неравенства. М.: Наука, 1968, 488 с.

11. Карманов B. Г. Математическое программирование. М.: Наука, 1980, 256 с.

12. Боровков $A$. A. Математическая статистика. Оценка параметров. Проверка гипотез. М.: Наука, 1984, 472 c.

Поступила в редакцию

14.I.1997 